\title{
Power Forecasting of Combined Heating and Cooling Systems Based on Chaotic Time Series
}

\author{
Liu Hai, ${ }^{1,2}$ Song Yong, ${ }^{2}$ and Du Qingfu ${ }^{2}$ \\ ${ }^{1}$ School of Energy and Power Engineering, Shandong University, Jinan 250061, China \\ ${ }^{2}$ School of Mechanical, Electrical \& Information Engineering, Shandong University at Weihai, Weihai 264209, China \\ Correspondence should be addressed to Liu Hai; sdulh8691@163.com
}

Received 21 October 2014; Revised 2 May 2015; Accepted 4 May 2015

Academic Editor: Kalyana C. Veluvolu

Copyright (C) 2015 Liu Hai et al. This is an open access article distributed under the Creative Commons Attribution License, which permits unrestricted use, distribution, and reproduction in any medium, provided the original work is properly cited.

\begin{abstract}
Theoretic analysis shows that the output power of the distributed generation system is nonlinear and chaotic. And it is coupled with the microenvironment meteorological data. Chaos is an inherent property of nonlinear dynamic system. A predicator of the output power of the distributed generation system is to establish a nonlinear model of the dynamic system based on real time series in the reconstructed phase space. Firstly, chaos should be detected and quantified for the intensive studies of nonlinear systems. If the largest Lyapunov exponent is positive, the dynamical system must be chaotic. Then, the embedding dimension and the delay time are chosen based on the improved $\mathrm{C}-\mathrm{C}$ method. The attractor of chaotic power time series can be reconstructed based on the embedding dimension and delay time in the phase space. By now, the neural network can be trained based on the training samples, which are observed from the distributed generation system. The neural network model will approximate the curve of output power adequately. Experimental results show that the maximum power point of the distributed generation system will be predicted based on the meteorological data. The system can be controlled effectively based on the prediction.
\end{abstract}

\section{Introduction}

As the energy and environmental stresses increased, the distributed generation system has become the best energy supply options. The distributed generation system has been noted by many countries and regions as one of the first elements which saves primary energy and reduces greenhouse gas emissions [1]. It is a well-established technology with high efficiency and very low pollutant emissions. It achieves small installation space, low maintenance, and long life service. The system can allow a wide range of operating conditions to match thermal and electric end-user requirements. The building owner can invest in an on-site system to supply power using nonrenewable or renewable technologies. The on-site systems can also supply the heating and cooling integrated with heat exchangers, solar thermal collectors, and absorption chillers [2].

The operation and control of distributed generation system are facing a stringent challenge to keep the efficiency and stability of the system [3]. The major breakthrough has not been made in the effective solutions for optimal dispatch and control strategy of distributed generation system. On the other hand, the existence of the uncertainty in the supply available from renewable generators has caused severe difficulty for the control and optimization of the system. The distributed generation system is a very complicated nonlinear system with time varying. Chaos is an inherent property of nonlinear dynamic system. Therefore, the kinetic forms of distributed generation system must be chaotic. The forecasting of chaotic time series is widely applied in many fields [4]. The intention of chaotic time series forecasting is to establish a nonlinear model of the dynamic system based on real time series in the reconstructed phase space. The most common methods of chaotic time series forecasting mainly include global forecasting method [5], local-region forecasting method [6], self-adapting forecasting method [7], and Lyapunov exponent forecasting method [8].

The output power of the distributed generation system and the environmental meteorology are strongly coupled. The chaotic time series of the microenvironment meteorological data and the output power are obtained by analysis of the previous meteorology rules in this region. In this paper, we build the model of the microenvironment meteorology and 


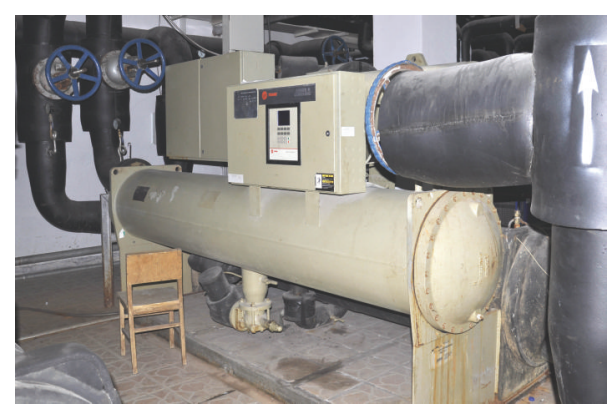

(a) The cooling subsystem

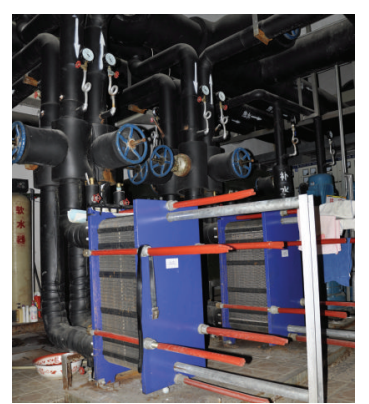

(b) The heating subsystem

Figure 1: The combined heating and cooling system.

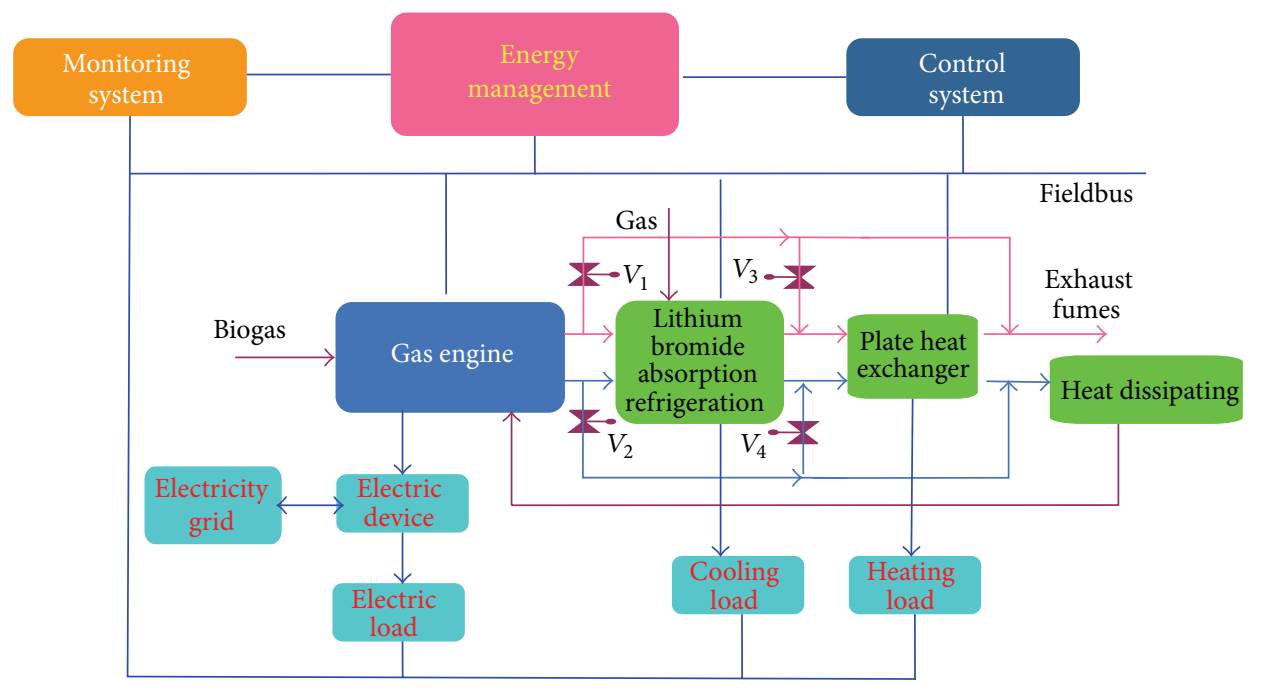

FIGURE 2: The block diagram of distributed generation system.

the output power of the distributed generation system based on neural network and chaos time series forecasting. The neural network is trained based on the training samples, which are chaotic time series obtained from the distributed generation system. The neural network approximates the curve of output power adequately. The functional relationships between the output power and the meteorological data are fitted to build the chaos forecasting model of the output power. The maximum power point is tracked by the chaos forecasting model in the conversion process between the renewable energy sources and the conventional energy sources. Then, different kinds of energy system are controlled harmoniously and banded together closely. The model of the system is mostly established based on accurate data. The error is general among experimental data owing to the disturbance. The precision of forecasting model based on neural network depends on the generalization of neural network. In order to improve the neural network predictor, the structure and parameters of the model should be optimized.

\section{The Distributed Generation System}

With the increasing demand for energy, the shortage of energy sources in the world is becoming more and more serious. At the same time, the consumption of the traditional energy sources aggravates the environmental pollution. Therefore, the utilization and the critical technology of renewable energy source have been paid more and more attention. The combined cooling, heating, and power systems have been considered as the best solution of future energy supply $[9,10]$. This paper deals with the power forecasting of renewable distributed energy system as depicted in Figure 1. The distributed generation systems operate based on the idea of the comprehensive step utilization of different grades heat energy [11]. The operation of the system is the conversion process of different grades energy. The different subsystems are mutually interrelated and constrained during the operation of the system. So the operation of the distributed generation system is a complex process with many factors.

The distributed generation system can be conceptually seen as being composed of the combination of local subsystems producing electricity, heat, cooling, and so on. Figure 2 shows the basic layout with the relevant energy flows. The core of the system is represented by one chemical block and two main physical blocks: the biomass gas generating system, the lithium bromide absorption refrigeration cycle, and the plate heat exchanger. 
The chemical reaction is the process in which biomass gas is converted to electrical energy in the biomass gas generating system. The available solid biomass resources with low calorific value are convertible to gas. Small electricity generators are powered by biogas or natural gas. As one bioenergy technology, biomass gas generating technology can provide clean energy. The biomass gas generating system consumes large amounts of solid reject and generates enormous amounts of thermal and electrical energy. The waste heat of the generating unit is used to drive the lithium bromide absorption refrigeration cycle and the plate heat exchanger.

The heat exchanging processes are physical reactions. The refrigerant vapour is generated in evaporator of the lithium bromide absorption refrigerator. Then, the lithium bromide solution will become dilute because amount of vapour is absorbed by the solution in absorber. The dilute solution is sent back to the heat exchanger by the circulating pump. The solution is concentrated and further heated by steam or hot water in the generator. The temperature of cool dilute solution rises through heat exchanger between the generator and the absorber. The metal plates are used to transfer heat between two fluids in a plate heat exchanger. The lithium bromide solution and refrigerant water are fed into tubes of the plate heat exchangers. The system also includes monitoring system, energy management system, and control system. The safe and stable running of the distributed generation system is ensured.

The powers of different subsystems are all subject to the fluctuation of the external environmental state, meaning the renewable energy is intermittent. The efficient control is a serious challenge in long cycle, security, and steady running. Therefore, it is very necessary for the prediction research of the renewable energy system. The real information can be supplied to distribution system if applicable. The short-term power forecasting can improve system operating efficiency and flexibility.

\section{Phase Space Reconstruction of Chaotic Time Series}

Generally, only a single scalar time series can be measured from a real physical system. So the phase space reconstruction from time series is the first stage in nonlinear time series analysis of data from chaotic systems [12]. All possible state variables cannot usually be obtained by the observation of a real process. Either not all state variables are measured or not all of them can be known. However, due to the couplings between the components of a chaotic system, we can reconstruct a phase space from a single observation by a time delay embedding [13].

Supposing that a continuous time dynamical system is described as follows:

$$
\frac{d(x)}{d t}=F(x(t)), \quad x \in R^{n},
$$

where $x$ is the state variable and $F$ is the kinetic equation of the system, the continuous system will become a discrete system after discretization:

$$
x(t+1)=f(x(t)) ; \quad t=0,1, \ldots ; R^{n} \longrightarrow R^{m},
$$

where $x$ is discrete state variable and $f$ is discrete kinetic equation. According to Taken's embedding theorem, some state variants of the original system can be analyzed by observing the system state $x(t)$. The most common technique is time delay method for phase space reconstruction. A scalar time series $x_{i}(t)$, where $i=1,2, \ldots, N$, can be embedded into an $m$-dimensional space to represent the dynamic system as follows:

$$
X(t)=\{x(t), x(t-\tau), \ldots, x(t-(m-1) \tau)\},
$$

where $t=1,2, \ldots, m, m$ is embedding dimension, and $\tau$ is the delay time.

The phase space reconstruction system is an orthogonal projection from $p$-dimensional space to $m$-dimensional subspace. The inherent evolution law of the dynamic system can be described in the form of an $m$-dimensional mapping. The linear smooth mapping $M$ is defined as follows:

$$
X(t+h)=M\{x(t), x(t-\tau), \ldots, x[t-(m-1) \tau]\},
$$

where $h$ is the prediction time domain.

The forecasting model is derived from the expression of the mapping equation (see (4)). But it is difficult to introduce the analytical expression in practice. The forecasting model based on neural network can be trained by training samples such as $[X(t), X(t+h)]$. The neural network model can approximate the linear smooth mapping $F$ adequately.

The delay time and embedding dimension play an important role in the phase space reconstruction according to a scalar time series. However, it is often extremely difficult to choose the parameters such as embedding dimension and delay time for phase space reconstruction. Normally, the delay time and embedding dimension are chosen independently. However, in recent years, some researchers contend that the delay time and embedding dimension are interrelated; that is, the delay time window should be estimated firstly for the choice of delay time and embedding dimension [14]. The delay time window can be ascertained using C$\mathrm{C}$ method. However, the exact selection of delay time can only be practicable by conventional C-C method. This paper tries to use the improved $\mathrm{C}-\mathrm{C}$ method to select embedding window [15]. The embedding window is first estimated based on the mean correlation integral. Then, the delay time can be chosen according to the conventional C-C method. The embedding dimension can be obtained according to the relation between embedding window and delay time. Owing to the mean correlation integral with a clear physical meaning, the optimal embedding window can be estimated objectively. In accordance with the above method, the parameters of phase space reconstruction can be estimated accurately.

\section{Echo State Network}

Echo state network (ESN) is a new-style recurrent neural network with a sparsely connected hidden layer $[16,17]$. The weights and connectivity of hidden layer are randomly assigned and are fixed. The weights of output layer can be learned from training samples. The discrete-time neural network with $K$ input units, $N$ internal network units, and $L$ 


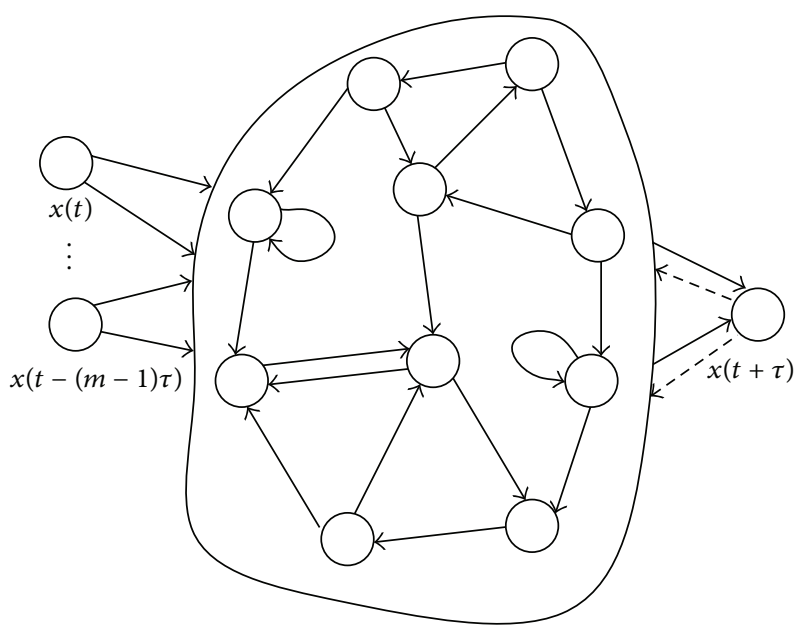

FIgURE 3: Architecture of echo state network.

output units is governed by the following state updating equation:

$$
x(n+1)=f\left(W x(n)+W_{\text {in }} u(n+1)+W_{f b} y(n)\right),
$$

where $u(n), x(n)$, and $y(n)$ denote the activations of input units, internal units, and output units at time step $n$, respectively. Furthermore, $u(n)$ is a $K$-dimensional input signal, $u(n)=\left(u_{1}(n), \ldots, u_{K}(n)\right), x(n)$ is a $N$-dimensional internal state, $x(n)=\left(x_{1}(n), \ldots, x_{N}(n)\right)$, and $y(n)$ is a $L$-dimensional output signal, $y(n)=\left(y_{1}(n), \ldots, y_{L}(n)\right), f$ is a sigmoid function (usually the logistic sigmoid or the tanh function), $W$ is the reservoir weight matrix, $W_{\text {in }}$ is the input weight matrix, and $W_{f b}$ is the output feedback weight matrix. The output of the neural network can be obtained according to the following equation:

$$
y(n+1)=f_{o}\left(W_{o}(x(n+1))\right),
$$

where $f_{o}$ is the activation functions of output units, $x(n+1)$ and $y(n+1)$ are the internal activation vectors and the output activation vectors at time step $n+1$, respectively, and $W_{o}$ is the output weight matrix. Figure 3 shows the basic network architecture.

The training of echo state network can be done in two stages: sampling and weight computation. During the sampling stage of the training, the echo state network is driven by the input sequence $u(n)$, which yields the internal states $x(n)$. The outputs of echo state network can be obtained using the system equations (5)-(6). The desired outputs $d(n)$ are written into the output units and pumped into the internal states through the back projection connections. The internal states $x(n)$ are collected into a state collection matrix M. Likewise, the teacher outputs $d(n)$ are collected into the rows of a teacher output collection matrix $\mathbf{T}$. The teacher signal series $d(n)$ is approximated as a linear combination of the internal states $x_{i}(n)$ by the following equation:

$$
d(n) \approx y(n)=\sum_{i=1}^{n_{o}} w_{i}^{o} x_{i}(n),
$$

where $n_{o}$ is the number of the output weights and $w_{i}^{o}$ is the output weight corresponding to internal state $x_{i}(n)$.

The mean squared training error is defined as

$$
\operatorname{MSE}=\frac{1}{N} \sum_{n=1}^{N}\left(d(n)-\sum_{i=1}^{n_{o}} w_{i}^{o} x_{i}(n)\right)^{2},
$$

where $N$ is the time steps, for which the teacher signal is written into the output unit. The output weight matrix $W_{o}$ can be computed such that the mean squared training error MSE is minimized. Therefore, the optimization of output weight matrix is to minimize the objective function to make neural network approximate the mapping from input to output. The computation of output weight matrix is a linear regression problem. The solution of output weight matrix is ill-posed by linear regression algorithm. The desired output weight matrix which minimizes MSE can be obtained by multiplying the pseudoinverse of $\mathbf{M}$ with $\mathbf{T}$ :

$$
W_{o}=M^{-1} T
$$

where $\mathbf{M}$ is the state collection matrix and $\mathbf{T}$ is the teacher output collection matrix. Then, the output weight matrix can be obtained based on the ready-made functions in Mathematica or Matlab. The optimal output weight matrix with minimized training error is implemented in echo state network. Now, the neural network predictor is ready for forecasting of the input-output time series.

\section{Forecasting Algorithm Based on Echo State Network}

Nonlinear time series forecasting is an important portion of current science and technology [18]. Generally, it is very difficult to obtain the mathematical models of complex dynamic systems. The system parameters such as input, output, and internal states are only measured. The univariate time series of a special parameter of the system can be established. The prediction of time series is very often generated by nonlinear forecasting models. The very common forecasting models are based on the chaos models or neural network models.

The forecasting methods of nonlinear time series include indirect forecasting and direct forecasting. The method of single-step forecasting has higher accuracy in practical applications. The direct multisteps forecasting model is concerned with the estimation of the system output at some time steps based on the mapping of input and output. There is no accumulation error for direct forecasting algorithms without the feedback of errors. However, with the increase of time steps, the forecasting model will be more complex. The singlestep forecasting model can carry out indirect multisteps forecasting through iterations. But the error of forecasting model accumulates along with time. The method of direct multisteps forecasting is applied to forecast the time series of distributed generation system power in this paper. A detailed description of each implement step of the forecasting algorithm is as follows:

(1) The chaotic analysis: calculate the value of the largest Lyapunov exponent to check whether the scalar time 
series is chaotic. Positive largest Lyapunov exponent shows the dynamic system is chaotic.

(2) The selection of system parameters: the embedding dimension $m$ and the delay time $\tau$ are chosen based on the improved C-C method.

(3) Phase space reconstruction: reconstruct an $m$-dimensional phase space from a single observation by a time delay embedding based on the delay time and embedding dimension.

(4) The training samples: the input vectors are $m(k)$, where $k$ is the number of training samples. The inputoutput pairs $\{m(k), X(k+h)\}$ are the training samples, which are obtained according to the prediction time domain $h$.

(5) The initialization of the forecasting model: the forecasting model based on ESN is initialized according to the learning algorithm of ESN. The output weights matrix is calculated based on (9).

(6) Prediction of the observation: the prediction value of energy in time domain $h$ can be obtained based on the real time observation of the system.

\section{Simulation and Analysis}

The equations of the distributed generation system are normally unknown. The interaction among the subsystems can be analyzed based on the dynamics system theory. The system law in the multidimensional phase space will be investigated based on the evolution track of the maximum power. During the operation of the distributed generation system, the maximum power was recorded every day. We gained a rich supply of data which would normally be accessible. In this paper, we collected 1000 data points, including 800 training datasets and 200 test data sequences. The data collection system consists of two parts, data acquisition and normalization processing. The process of data normalization can suppress the electromagnetic interferences and improve the generalization ability of neural network model. The method of normalization processing is provided. The equation of linear transformation can be represented as shown below:

$$
\bar{P}_{i}=\frac{P_{\max }-P_{i}}{P_{\max }-P_{\min }} \quad(i=1,2, \ldots),
$$

where $P_{\max }$ is the maximum of the power sequence, $P_{\min }$ is the minimum of the power sequence, and $P_{i}$ is the maximum power on the $i$ th day.

6.1. The Choice of Experimental Parameters. The problem of detecting and quantifying chaos is a key step for the intensive studies of nonlinear systems. The Lyapunov exponents are the important indexes to quantify the sensitivity on initial conditions for a dynamical system. If the dynamical system is chaotic, at least one Lyapunov exponent must be positive. Therefore, we only need to calculate the largest Lyapunov exponent to judge the chaotic characters. The largest Lyapunov exponent characterizes the rate exponential divergence of the nearby trajectories in the reconstructed phase
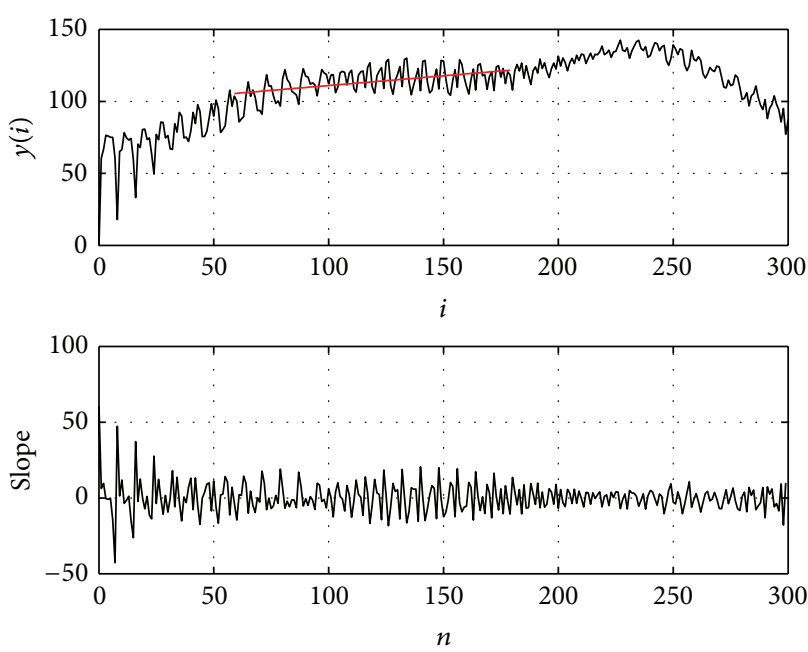

FIgURE 4: The largest Lyapunov exponent.

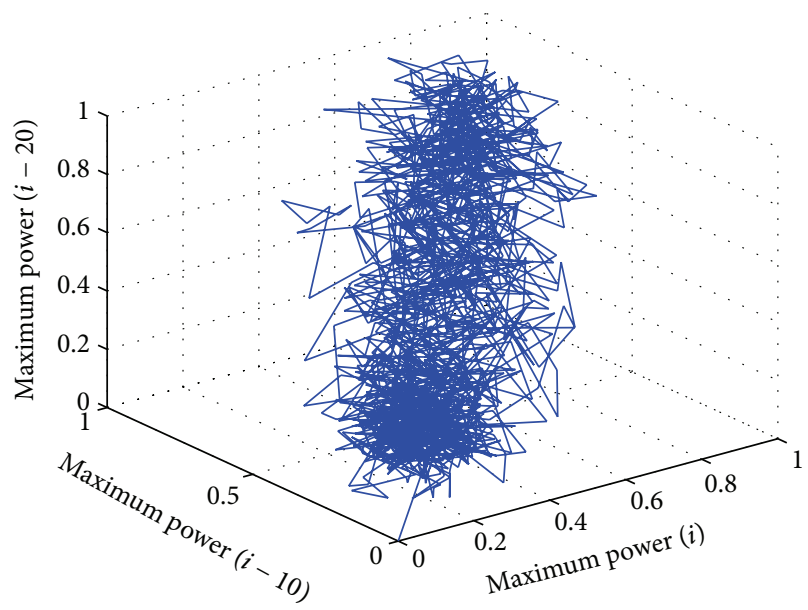

FIGURE 5: The attractor of chaotic power time series.

space. There are also lots of algorithms to estimate the largest Lyapunov exponent of an experimental time series. In this paper, the largest Lyapunov exponent was calculated with small amount of data. The estimate of the largest Lyapunov exponent is $0.13 \mathrm{bit} / \mathrm{s}$ (shown in Figure 4).

The embedding dimension $m$ and the delay time $\tau$ are chosen based on the improved C-C method. Then, we obtain embedding dimension $m=10$ and delay time $\tau=8$. The phase space is reconstructed based on the embedding dimension and the delay time. The attractor of chaotic power time series is shown in Figure 5. In practice, there will always be some form of corrupting noise. Thus, any real time series, even if mostly deterministic, will contain some randomness. The evolution of the distributed generation system being chaotic means that every point in the reconstructed space is approached arbitrarily but not coincident on the pseudo periodic orbits.

6.2. The Analysis of Simulation Results. The neural network model is trained based on the training samples. The teacher 


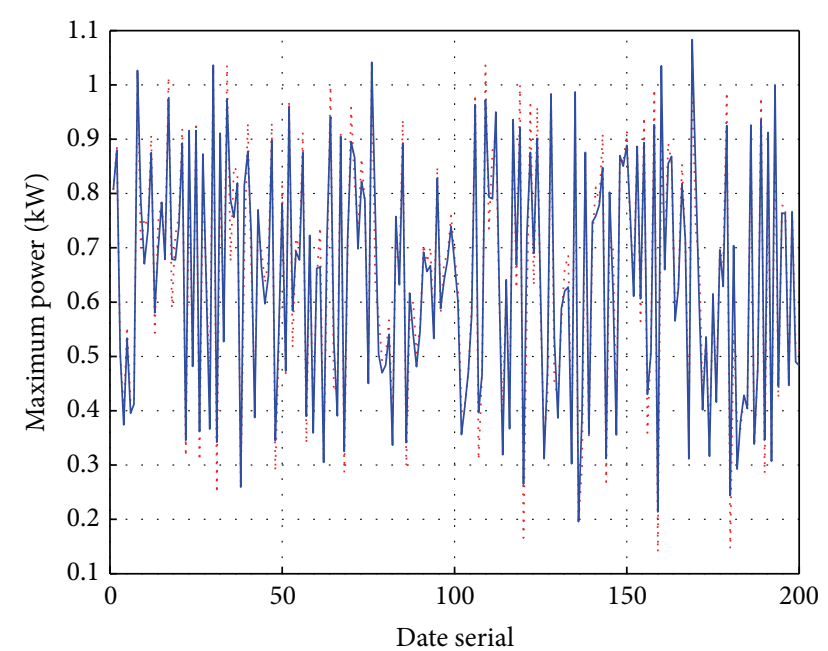

…. Observed power

— Predicted power $(\mathrm{kW})$

FIGURE 6: The estimated output and observations.

signal is written into the output unit for times $n=1, \ldots, 800$. The internal states $x(n)=\left(x_{1}(n), \ldots, x_{20}(n)\right)$ for $n=$ $1, \ldots, 800$ are collected as a row of a state-collecting matrix $\mathbf{M}$ of size $800 \times 20$ during the sampling period. At the same time, the teacher outputs $d(n)$ are collected into a row of a matrix T of size $800 \times 1$. Then, we can compute output weights $w_{o}$ for linear output unit $y(n)$ such that the teacher time series $d(n)$ is approximated as a linear combination of the internal activation time series $x_{i}(n)$ by (9). The multistep prediction can be obtained with testing samples. The predictor can forecast multistep value directly with different time domain.

The result of direct prediction based on echo state network for the maximum power series of the distributed generation system is shown in Figure 6. The solid line denotes the predicted value; the dotted line is for the observed value. We observe that the prediction model based on echo state network has high accuracy in direct prediction owing to the coincidence of prediction and actual curve. The max prediction error is about 0.17 (shown in Figure 7). Figure 8 is the error of the traditional multilayer perceptron. The predictor based on echo state network has higher accuracy.

\section{Conclusion}

The microenvironment meteorological data are coupled with the output power of the distributed generation system. Theoretical proof and experimental results show that the output power of the distributed generation system is chaotic time series. The prediction of chaotic time series is to establish a nonlinear model of the dynamic system based on real time series in the reconstructed phase space. Firstly, we should detect and quantify chaos for the intensive studies of nonlinear systems. If the largest Lyapunov exponent is positive, the dynamical system must be chaotic. Secondly, the embedding dimension and the delay time are chosen based on the improved C-C method. The attractor of chaotic power time

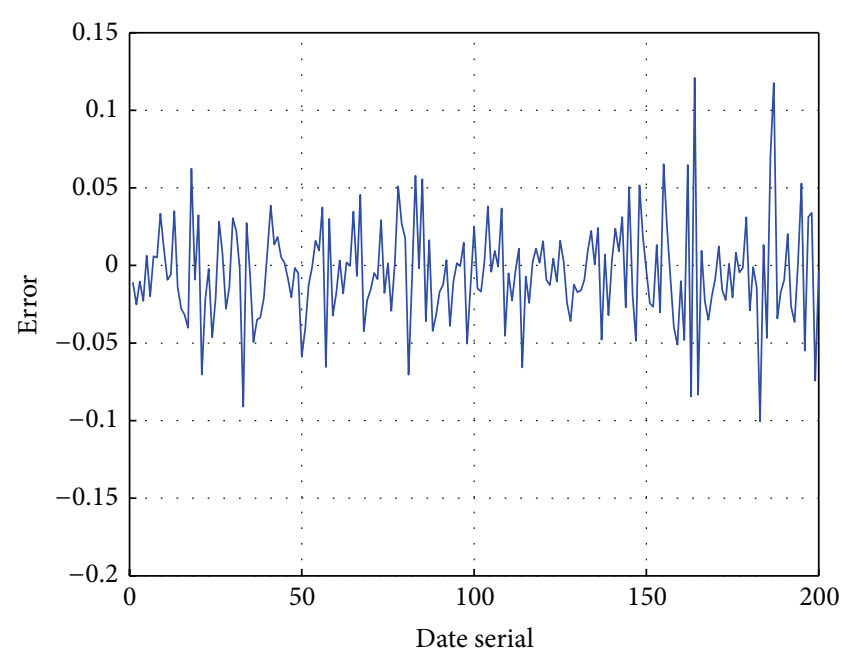

FIGURE 7: The error of the predictions.

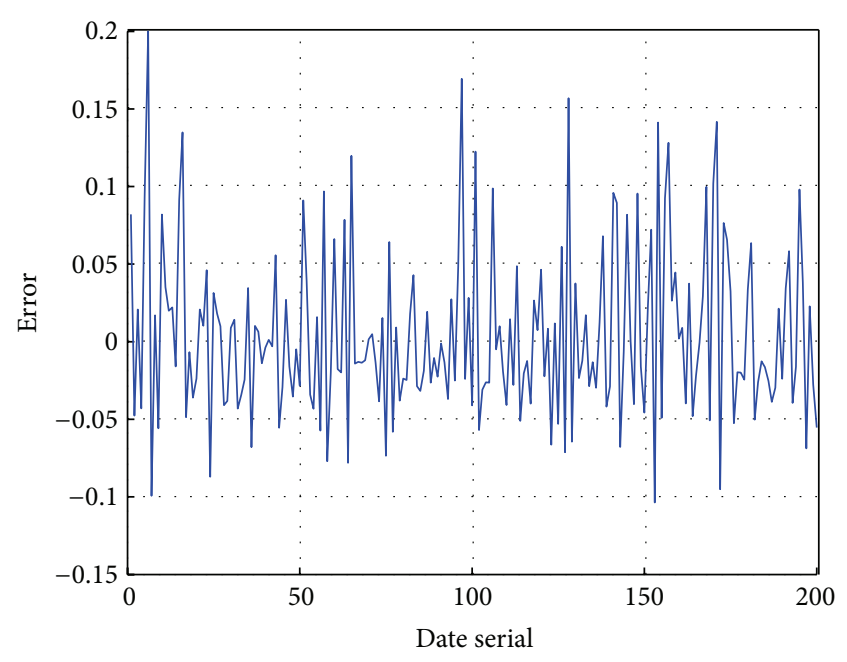

FIGURE 8: The error of the traditional multilayer perceptron.

series can be reconstructed based on the embedding dimension and delay time in the phase space. Lastly, the neural network model is trained based on the training samples, which are obtained from the distributed generation system. The neural network will approximate the curve of output power adequately. The maximum power point of the distributed generation system will be tracked based on the meteorological data.

The measuring errors are unavoidable among experimental data due to the disturbance and many other factors. The prediction precision of the neural network model is largely dependent on the generalization of neural network. Therefore, we should optimize the structure and parameters of the model for better performance. Experimental results show that the prediction model based on echo state network has high accuracy. The chaotic prediction model can approximate the mapping between the maximum power point of the distributed generation system and the meteorological data. The echo state network model can make rather remarkably 
accurate predictions about the maximum power of the distributed generation system.

\section{Conflict of Interests}

The authors declare that there is no conflict of interests regarding the publication of this paper.

\section{Acknowledgments}

This work is supported by the National Natural Science Foundation of China (nos. 61473174, 61105100, and 51376110), the Specialized Research Fund for the Doctoral Program of Higher Education (no. 20130131130006), Project funded by China Postdoctoral Science Foundation (no. 2014M551907), and Independent Innovation Foundation of Shandong University (no. 2013ZRQP002).

\section{References}

[1] G. Angrisani, C. Roselli, and M. Sasso, "Distributed microtrigeneration systems," Progress in Energy and Combustion Science, vol. 38, no. 4, pp. 502-521, 2012.

[2] K. A. Pruitt, S. Leyffer, A. M. Newman et al., Optimal Design and Dispatch of Distributed Generation Systems, Argonne National Laboratory, Chicago, Ill, USA, 2012.

[3] G. Pepermans, J. Driesen, D. Haeseldonckx, R. Belmans, and W. D’haeseleer, "Distributed generation: definition, benefits and issues," Energy Policy, vol. 33, no. 6, pp. 787-798, 2005.

[4] Y. Song, Y. Li, Q. Wang, and C. Li, "Multi-steps prediction of chaotic time series based on echo state network," in Proceedings of the IEEE 5th International Conference on Bio-Inspired Computing: Theories and Applications (BIC-TA '10), pp. 669672, IEEE, Changsha, China, September 2010.

[5] S. Michanos, A. Tsakoumis, P. Fessas, S. Vladov, and V. Mladenov, "Short-term load forecasting using a chaotic time series," in Proceedings of the International Symposium on Signals, Circuits and Systems (SCS '03), pp. 437-440, Iasi, Romania, 2003.

[6] X. An, D. Jiang, M. Zhao, and C. Liu, "Short-term prediction of wind power using EMD and chaotic theory," Communications in Nonlinear Science and Numerical Simulation, vol. 17, no. 2, pp. 1036-1042, 2012.

[7] C. Qin, C.-C. Chang, and L.-T. Liao, "An adaptive predictionerror expansion oriented reversible information hiding scheme," Pattern Recognition Letters, vol. 33, no. 16, pp. 2166-2172, 2012.

[8] J. Zhang, K. C. Lam, W. J. Yan, H. Gao, and Y. Li, “Time series prediction using Lyapunov exponents in embedding phase space," Computers \& Electrical Engineering, vol. 30, no. 1, pp. 115, 2004.

[9] J. M. Guerrero, F. Blaabjerg, T. Zhelev et al., "Distributed generation: toward a new energy paradigm," IEEE Industrial Electronics Magazine, vol. 4, no. 1, pp. 52-64, 2010.

[10] G. Chicco and P. Mancarella, "Distributed multi-generation: a comprehensive view," Renewable and Sustainable Energy Reviews, vol. 13, no. 3, pp. 535-551, 2009.

[11] G. Abdollahi and M. Meratizaman, "Multi-objective approach in thermoenvironomic optimization of a small-scale distributed CCHP system with risk analysis," Energy and Buildings, vol. 43, no. 11, pp. 3144-3153, 2011.
[12] D. Xia, S. Song, J. Wang, J. Shi, H. Bi, and Z. Gao, "Determination of corrosion types from electrochemical noise by phase space reconstruction theory," Electrochemistry Communications, vol. 15, no. 1, pp. 88-92, 2012.

[13] Z. Xie and K. Wang, "Selection of embedding parameters in phase space reconstruction," in Proceedings of the 2nd International Conference on Intelligent Computing Technology and Automation (ICICTA '09), pp. 637-640, IEEE, Hunan, China, October 2009.

[14] W.-D. Cai, Y.-Q. Qin, and B.-R. Yang, "Selection of delay time window and delay time in phase space reconstruction," in Proceedings of the International Conference on Computational Intelligence and Security (CIS '07), pp. 526-530, December 2007.

[15] Y.-B. Li, Y. Song, and C.-H. Li, "Selection of parameters for phase space reconstruction of chaotic time series," in Proceedings of the IEEE 5th International Conference on Bio-Inspired Computing: Theories and Applications (BIC-TA '10), pp. 30-33, IEEE, Changsha, China, September 2010.

[16] J. Herbert, Tutorial on Training Recurrent Neural Networks, Covering BPPT, RTRL, EKF and the "Echo State Network" Approach, GMD-Forschungszentrum Informationstechnik, Bremen, Germany, 2002.

[17] H. Jaeger, M. Lukoševičius, D. Popovici, and U. Siewert, "Optimization and applications of echo state networks with leakyintegrator neurons," Neural Networks, vol. 20, no. 3, pp. 335-352, 2007.

[18] K. A. Bredahl and T. Timo, Forecasting with Nonlinear Time Series Models, School of Economics and Management Aarhus University, Aarhus, Denmark, 2011. 

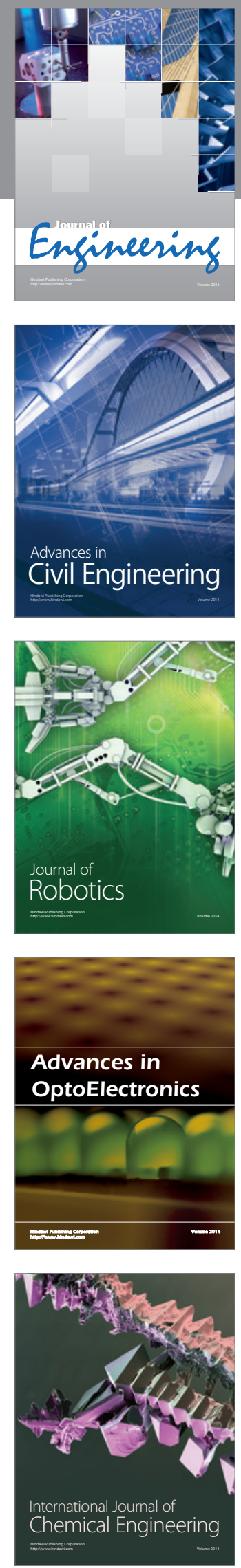

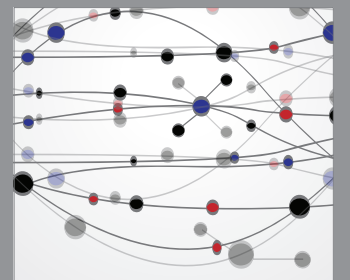

The Scientific World Journal
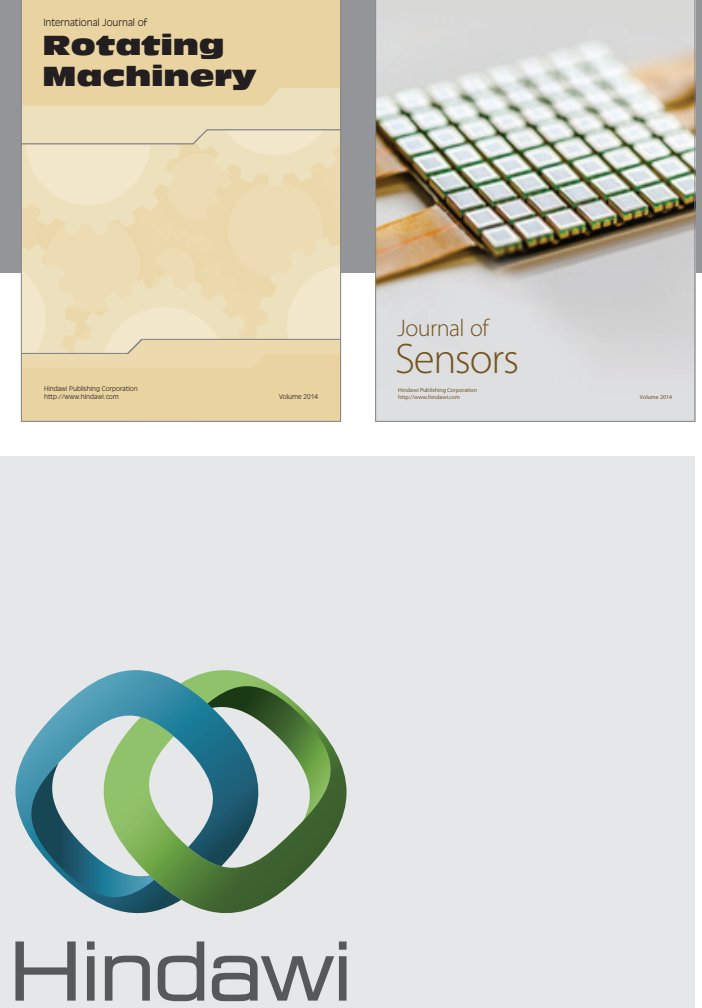

Submit your manuscripts at http://www.hindawi.com
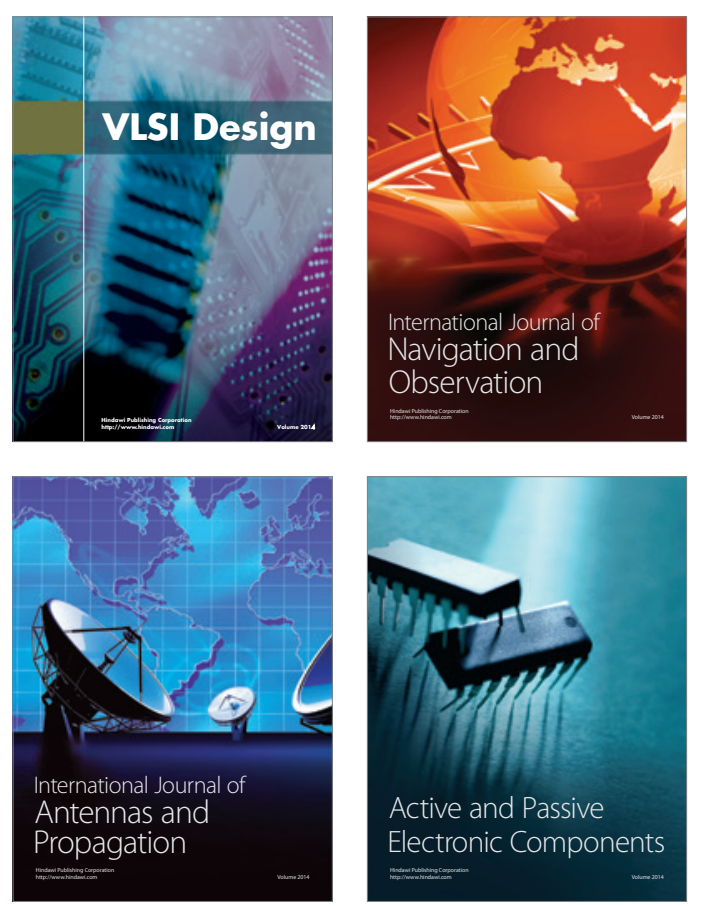
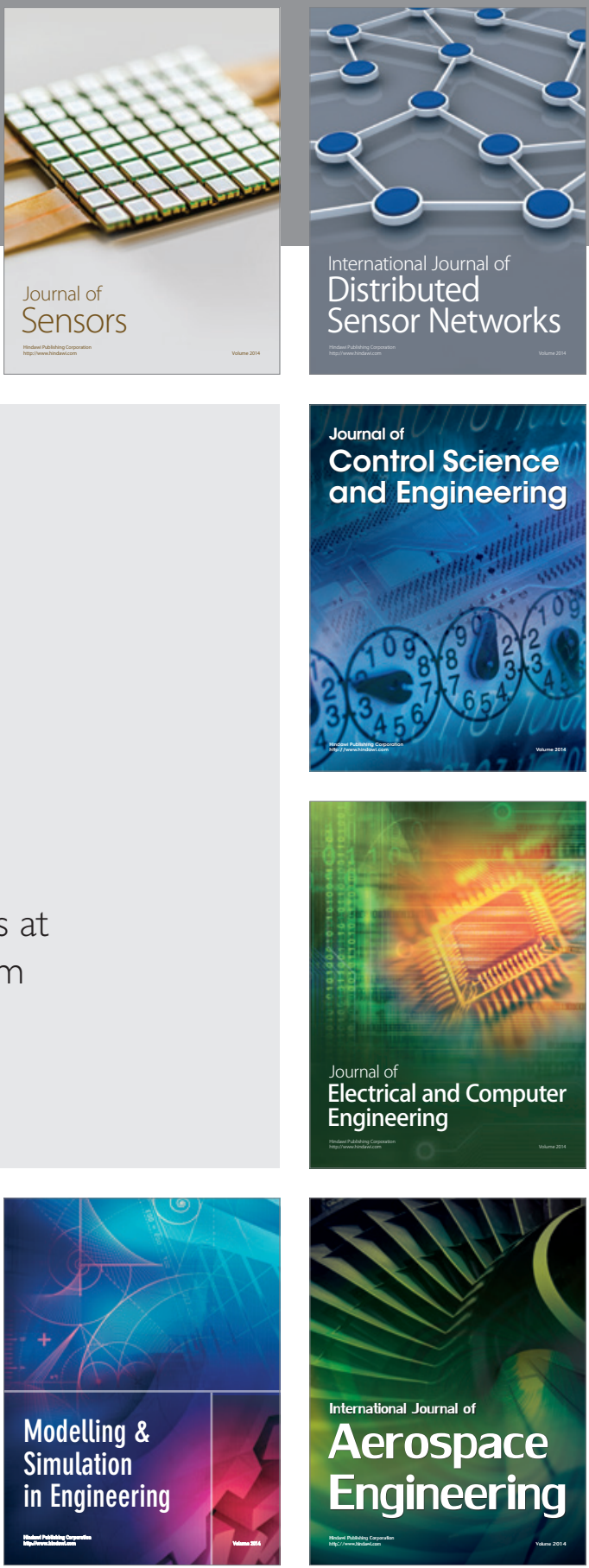

Journal of

Control Science

and Engineering
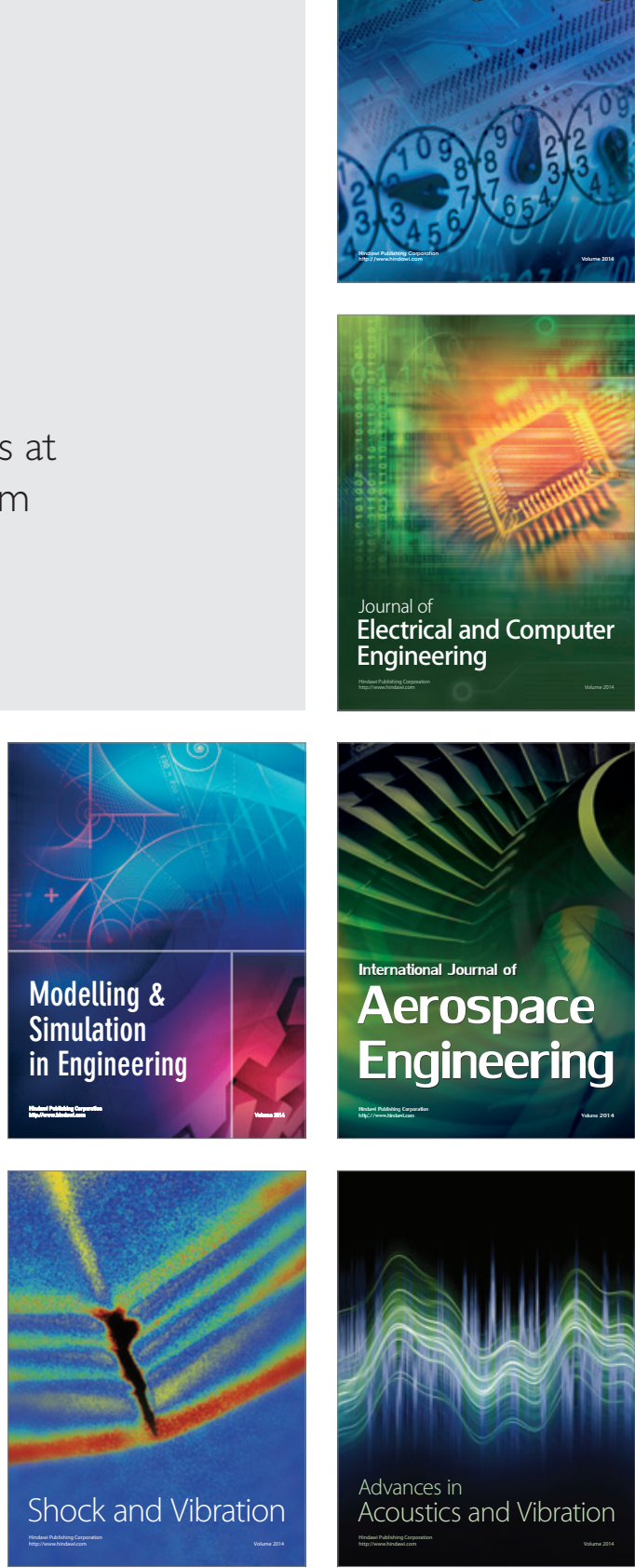